\title{
WORKFORCE Clinical supervision of physician associates (PAs) in primary care: who, what and how is it done?
}

\author{
Authors: Ria Agarwal ${ }^{A}$ and Julie Hoskin ${ }^{B}$
}

The physician associate (PA) role is gaining momentum as a healthcare professional who supports medical workload in primary care, yet there is a lack of clinical literature around how best to clinically supervise this new role. This seems especially pertinent amid the recent funding initiatives that encourage employment of PAs to aid the increasing demands in primary care, especially with the added stressors of the COVID-19 pandemic. There is a need for clinical supervisors to be aware of what their responsibilities are when employing and supervising a PA. Qualitative feedback from a cohort of primary care PAs in Sheffield alongside the authors' own expertise have been collated to produce recommendations to supplement existing documentation from the Faculty of Physician Associates. The paper seeks to rapidly initiate a starting point in clinical literature around the breadth of considerations within PA supervision. These recommendations include, but are not limited to, a discussion at the onset of PA employment of mutual needs and a specified supervisory schedule, alongside named clinicians who generally address clinical and pastoral components periodically. This accompanies an induction into the practice and general clinical support that is initially more intensive but otherwise remains available when the PA feels it is required.

KEYWORDS: physician associate, general practice, allied healthcare professional, clinical supervision, GP PA

DOI: 10.7861/fhj.2020-0241

\section{Introduction}

Physician associates (PAs) remain relatively new entrants in the healthcare workforce but numbers are steadily increasing' especially with strategies to incentivise this further (for example, the recent Direct Enhanced Service (DES) contract for Additional Roles

Authors: ${ }^{\text {A }}$ physician associate, Sothall Medical Centre, Sheffield, UK, senior lecturer, Sheffield Hallam University, Sheffield, UK and allied health professional representative, Royal College of General Practitioners South Yorkshire, North Trent Faculty, Sheffield, UK;

${ }^{B}$ advanced nurse practitioner and physician associate preceptorship lead, Sheffield Clinical Commissioning Group, Sheffield, UK
Reimbursement Scheme). ${ }^{1,2}$ There is a steep learning trajectory for PAs from being a postgraduate student for 2 years to then assessing undifferentiated patients with some degree of autonomy and responsibility. As with any healthcare professional, the first few years are vital in shaping them clinically, in addition to shaping their views of a career in primary care. Unlike the rest of the medical workforce, PAs are uniquely able to move between primary and secondary care, which, in the future, may lead to retention issues if working in primary care leads to dissatisfaction. In our experience locally and regionally, a lack of clear guidance has led to differences in clinical supervision of primary care PAs, which can lead to PA dissatisfaction, and, in one instance known to us, a preceptee's failure. These issues, in addition to the need to continuously ensure patient safety, have motivated us to investigate this and rapidly initiate a starting point in clinical literature around the breadth of considerations within PA supervision. There is a need to establish guidance around clinical supervision offered by employers, who may also be new to PAs themselves and juggling recruitment or management of these additional roles as part of a strategy to handle the increased clinical workload due to COVID-19. ${ }^{3}$

\section{The who and the what}

The term 'clinical supervision' can be interpreted in several ways, and it is often done so differently by different professional bodies. ${ }^{4}$ In general practice (GP) vocational training schemes, the role of the clinical supervisor overseeing the trainees' daily caseload is distinct from that of the educational supervisor, who looks at the generalised trajectory and progress of the trainee. For many years in nursing, 'clinical supervision' referred to a supportive peer-based professional relationship between colleagues, sometimes outside of each other's organisations. More recently this has become more analogous with the term 'mentor', which overlaps somewhat with the mentorship stream within the Sheffield PA preceptorship scheme. Funding from Sheffield Clinical Commissioning Group (CCG) following a signed supervision agreement enables clinical supervision in-house to be supplemented by mentorship from an external senior PA, alongside protected weekly educational sessions for preceptees. Work by Hoskin et al indicates this was well received by preceptees. ${ }^{5}$

For PAs based in regions lacking external support from CCGs or Health Education England (HEE), there may be more pressure on the employer to perform these different aspects of educational and clinical supervision. While PAs are trained to the medical model, they are not doctors, so even an experienced GP trainer 
may not fully be aware of the needs of the PA. The employer guidance provided by the Faculty of Physician Associates details a possible induction package for a GP supervisor and PA, but the components of supervision are less well established. ${ }^{6}$ Despite following the same national curriculum, some newly qualified PAs may be confident from the outset, whereas others may require more nurturing. Regarding the format of supervision, some PAs may respond better to a 'buddy' approach as and when required, while others may benefit from formal discussions. In order to fulfil the expectations of both parties, it is recommended that a discussion of the content and frequency of clinical supervision takes place early in PA-supervisor discussions. An unhappy supervisor who has misunderstood either the requirement of their role, or the PA role itself, will probably find the relationship as unsatisfactory as the PA. By preparing both the practice and the PA for the role, failure and dissatisfaction from either party is much less likely.

\section{Methodology}

Using local practice as a starting point, a qualitative online questionnaire was sent to 20 primary care PAs with varying lengths of time in post within 16 practices in Sheffield and the surrounding areas, enquiring about the frequency and nature of clinical supervision received in-house in their first year of practice (Box 1). 14 anonymised responses were collated with the authors combined experiences of educating, working as or alongside PAs, to investigate what supervision was provided for these PAs within their first year of primary care and gauge their level of satisfaction with this. The questions were focused around induction length and quality, clinical day-to-day supervision quantity and perceived satisfaction, and the presence of more formal periodic supervisor meetings. The questionnaire was designed to allow open-ended free-text responses regarding each of these areas. While this method traditionally has limitations, in this case it enabled us to have an exploratory approach to issues and ideas raised by each PA. A further limitation of these data is the small sample size, which was deliberately limited to local PAs to encourage confidence due to existing relationships with this cohort. Limiting the cohort to this geographical area has enabled consistency in other variables, such as some employers having prior knowledge of PAs through direct or indirect involvement with the local higher education institutions (HEIs). In addition, most respondents were either historically, or at the time of enquiring, a recipient of the support provided by the Sheffield preceptorship scheme and/or its associated education stream. Four out of the 14 respondents were initially employed in band 7 roles, and the remainder were historically or currently in the band 6 Sheffield PA primary care preceptorship scheme, although two had been employed in secondary care prior to their preceptorship year. Some practices went on to employ a second and, in one case, a third PA, so some supervisors may have had the benefit of hindsight from

Box 1. Qualitative questionnaire sent to primary care physician associates in Sheffield and surrounding areas, $n=14$

\section{Clinical experience level of physician associate respondents}

$>$ In your first year of practice post-qualification, were you employed as a preceptorship physician associate at band 6 or in a permanent band 7 post?

> If you weren't employed in a preceptorship, do you think this would have been helpful (eg components of Sheffield preceptorship scheme are half day per week protected study time, access to PA-specific taught sessions +/- consultation observation from another PA).

$>$ If you moved to general practice from another specialty (ie it wasn't your first post), please also state this here too.

Induction period questions

$>$ Were you given an induction period to the practice before you were expected to start seeing patients (eg approximately $X$ days or weeks of observing colleagues)?

$>$ If so, please describe your induction period below; stating the duration, and if it felt adequate to your clinical needs? If inadequate, please detail why you have stated this.

$>$ If no induction was provided, do you think this would have been helpful, and why?

\section{Day-to-day supervision questions}

> When you first started seeing patients, how often did a supervisor support you in debriefing/overseeing your patients (eg once/twice daily; once/twice weekly)?

$>$ If applicable to your current stage of practice, how did this change throughout the year? Was any change in supervision at your request or theirs? Did this feel clinically appropriate and safe to you?

> Did the level of supervision you were given feel appropriate to your clinical needs, or did it ever feel too much/little? Could it have been enhanced through the addition of any other aspect?

\section{Formal supervisory meetings questions}

$>$ Aside from clinical supervision of patient consultations, did you ever have periodic meetings with a supervisor to discuss non-patient facing issues (eg salary, appointment lengths, feedback or integrating you into the team)?

$>$ If so, how frequently did these meetings occur and what did you discuss?

> If you did not have access to such formalised meetings, do you think this would have been helpful to you? Did you have a named supervisor?

> Regarding appointment length, specifically, did your supervisor(s) approach appointment timing with you? Did the pace of appointment time reduction feel comfortable for you?

Do you have any additional feedback/comments around in-house supervision you received in your first year of general practice? 
employment of their first PA, although all such PAs were invited to complete the survey. In addition, respondents had varying levels of experience, ranging between a few months to almost 3 years. One of the authors is a PA herself, and was employed in a band 7 role post-qualification in 2013 , in addition to jointly supervising PAs and PA students at her practice for some time.

\section{Results}

The feedback suggested the respondents were generally happy with the level of support given. Two of the 14 respondents identified issues that will be discussed below; both were employed prior to the COVID-19 pandemic and commenced on band 6 preceptorships. The main issues identified were insufficient induction, absence of structured support and inadequate communication between the GP supervisor and the PA.

\section{How is it done?}

\section{Induction}

In relation to induction, the respondents identified that an induction period of up to 2 weeks was helpful to integration within the practice, with one preceptorship PA stating that the 2 days they were given felt insufficient. One PA identified that they had started working in the practice in one of the newly developed assistant practitioner PA posts owing to the delay in sitting their national exam due to the COVID-19 pandemic, thus behaving as an extended induction prior to starting work in a preceptorship PA post. Another PA similarly replied that they were a PA student at the practice prior to their employment there, which significantly helped with understanding practice ethos. One of the authors was given a whole week induction prior to seeing patients, which she felt was helpful at familiarising herself with the personalities of the six GP partners at her surgery, in addition to understanding how the different clinical and administrative roles 'join up' in the overall care of the patients at that practice. The induction aided awareness of important logistical issues, such as familiarisation with the IT system and how to access a GP for prescriptions, the lack of which was previously noted to be a reason contributing to a PA not progressing down the proposed preceptorship scale. ${ }^{5}$ Consequently, in the recently introduced 'network PA' posts where one PA may work at multiple practices in their working week, an extended induction may need to be a consideration to allow time for the PA to understand how each practice's microsystems work. While our paper does not specifically investigate practice staff or patient perceptions of the PA role, one of the authors notes that an induction period was also helpful in ensuring that staff understood her scope of practice, and the announcement of her arrival to patients was facilitated via the release of an article to the widely read local newspaper alongside updating the practice website.

\section{Day-to-day supervision}

Degrees of clinical supervision varied between practices, but most respondents were happy with the level of clinical supervision received. This was commonly a once or twice daily debrief initially encompassing review of all patients and access to a duty doctor throughout their clinic when required. Over the course of a year, this progressed to a weekly debrief and then an 'as required' approach when the PA felt ready for this. Two of the PAs stated they were not given any set debrief time despite being on a preceptorship; consequently, one PA stated they felt like a burden if they did wish to discuss a patient, and the other PA utilised the on-call doctor much more frequently since it was the only available opportunity to have a senior opinion. Naturally this can lead to frustration from the busy on-call doctor who may well be juggling multiple duties. Two of the respondents felt that having fewer doctors supervising them day-to-day was helpful in providing consistent clinical advice, with more opinions being confusing. One of the authors felt that, throughout her first year, the varying clinical opinions helped build her foundation of knowledge in the 'art' of medicine.

One of the authors and one respondent had their first 2 weeks of patients physically reviewed by the duty clinician before the patient left the surgery, and this may well be a helpful strategy in enabling the PA-supervisor relationship to build. Respondents found it helpful to also have feedback on their documentation and referral letters when they first started practice. As discussed by Drennan et al, the supervisor could, at periodic intervals, review a sample of the PA's consultations not already brought to their attention in order to ensure the PA is seeking support appropriately, feeding forward to the PA as required and enabling a culture of supportive transparency that ultimately ensures patient safety. ${ }^{7}$ Similarly, as the PA increases in autonomy, it may be helpful to the evolving PA-supervisor relationship to gain a fuller perspective on what the PA is managing within their scope of practice. Annual appraisal may be a helpful time to conduct this

\section{Formal supervisory meetings}

Alongside clinical supervision, most respondents had structured support in the way of monthly meetings with a named supervisor or GP partner to discuss non-patient facing issues, such as opportunities for development and identifying additional duties for the PA. One respondent identified that this was a helpful avenue to discuss feedback from colleagues and patients about the PA's performance, which aided confidence building and job satisfaction. Those that did not have such structured meetings said they would have found this helpful. Another respondent stated that appointment time length was reduced without any communication, so utilising meetings to discuss this in the first instance would likely have been beneficial. One of the authors found that supervisor meetings throughout her first year, initially monthly and then slowly phasing out, were helpful in ensuring that both the clinical aspects and business aspects of the PA role were fairly addressed at appropriate intervals, namely daily debriefing focusing on clinical areas and monthly meetings focusing on appointment timings and workload discussion.

Just as a GP trainee has a named clinical supervisor, it was felt by the respondents that these periodic meetings with just one or two named clinicians would have been preferable for continuity. One respondent stated the lack of periodic meetings was compensated for by just having one supervisor. At least two surgeries were utilising PAs whom they'd employed for 2-3+ years to jointly supervise more junior PAs and/or PA students. As these supervising PAs are often experienced in role-specific issues and permanent members of the team, it may well be helpful for the practice to formally include them in a co-supervising capacity with a named GP to consequently enable continuity for the more junior PAs. A supervisory role also adds an extra dimension in lateral career progression for the established PA while increasing their 
responsibilities within the practice; the benefits of which have been discussed in previous work by Agarwal et al. ${ }^{8}$ However, caution would need to be taken to ensure that the supervising PA was working within their scope, and the GP partners were comfortable with this arrangement.

\section{Conclusion}

We applaud the literature over the years which has increased appetite in the PA role and likely played a part in its establishment. In our opinion, the next discussions need to be around supporting and supervising PAs appropriately. Currently there is little clinical literature on components of clinical supervision for the PA role specifically, within or outside of the UK. This paper is merely a starting point for discussion based on qualitative experience of a small cohort of PAs and the expertise of the authors. A webinar was organised in collaboration with the Royal College of General Practitioners South Yorkshire North Trent Faculty (SYNT) by one of the authors in October 2020 to address this further. In the authors' experience, the first few years of clinical practice are vital in influencing not only retention and job satisfaction but also clinical progression of the developing PA. This not only requires financial and clinical investment from the employer's perspective but may also aid the PA's sense of belonging and career satisfaction. ${ }^{9}$ These factors, alongside appropriate financial reimbursement, can only increase the likelihood of PA retention in primary care.

With the above in mind, we make the following five-point recommendations to ensure patient safety regardless of the PA's starting salary.

\section{Induction}

> Upon initiation of the PA in post, there should be an induction period of 1-2 weeks to allow the PA to settle into the practice and familiarise themselves with logistics within the practice, practice staff and practice ethos. This also serves as an excellent opportunity for practice staff to understand how the PA fits into the practice team, and to inform patients of the addition of this new healthcare professional.

\section{Day-to-day supervision}

> A discussion about the frequency and role of supervision should take place during induction to discuss each participant's understanding and requirements. This should be revisited and adjusted at intervals.

> The clinical supervisor should preferably be one or two named permanent clinicians who oversee and support the PA's progress throughout the first year. This may include PAs established within the practice. Day-to-day debriefing may be supported by other clinicians who are willing to supervise the PA above their own clinical workload.

> Clinical supervision length and frequency should aim to enable clinical confidence and growth in the individual PA(s) and whatever is necessary to aid development of the PA-supervisor relationship. In this particular study, the respondents found review of every patient initially, progressing throughout the first year to debriefing as required, was an effective model of clinical supervision.

\section{Formal supervisory meetings}

$>$ In tandem with discussion of clinical problems or formal teaching, there should be wider discussions at periodic intervals of the PA's progress, such as once per month initially. Within these meetings, general feedback and pastoral components should be addressed alongside appointment timings and workload discussions.

The findings of this small-scale research supplement existing documentation from the Faculty of Physician Associates and echo previous work by Kilminster et al particularly around the importance of structured supervision that addresses clinical and pastoral components. ${ }^{10}$ However, we also add that named supervisors having a clear understanding of what each party expects from the PA-supervisor relationship aids PA integration into the team and can only help PA clinical growth through confidence building. We feel the wrong approach is to have supervision perceived by the PA as inadequate in their transitional period of joining and becoming embedded into the practice, which must be a consideration in our even busier climate of the COVID-19 pandemic.

Looking to the USA where the profession originated, when the profession was new, PAs were legally required to work with a specific physician in order to practice. This has since been loosened through the passing of Optimal Team Practice which enables more collaborative working across the team. ${ }^{11}$ Some states specifically indicate a set number of hours per week or month of supervision and even a limit on PA supervisors, but factors including scope of practice of that PA and the ruralness of the specific location likely plays a part in this legislature. ${ }^{12}$ While clinical supervision details for newly qualified PAs otherwise do not appear to be explicitly defined in the USA, the role is more widely understood in lieu of the longevity of the role and significantly higher PA numbers (estimated to be over 140,000). ${ }^{13}$

\section{Summary}

\section{What is known?}

In our current COVID-19 climate, medical workload is high and, at the same time, PAs are increasingly being recruited into primary care due to the recent funding incentives. In order to successfully integrate PAs into the medical team, they need to be appropriately supported and supervised in order to maintain patient safety. This responsibility can fall to a GP who is experienced in training doctors or other professionals but may have little experience of the PA role itself. If not done appropriately, this can lead to PA job dissatisfaction or failure to clinically thrive.

\section{What is the question?}

How can clinical supervisors successfully support and supervise this new healthcare professional in primary care; what factors should be considered here? What are the main components of the role of a PA clinical supervisor in contrast to other healthcare professionals?

\section{What was found?}

Qualitative feedback from band 6 and 7 PAs in Sheffield demonstrated they felt well supported by their clinical supervisors on the whole. Those that did not feel supported stated this was due to an inadequate induction period, absence of structured support and/or debriefing from their supervisor, or inadequate communication from their supervisor. These recommendations support the clinical supervision stream identified in the previously proposed PA preceptorship scale. ${ }^{5}$ 


\section{What is the implication for practice now?}

Our proposed five-point recommendations include, but are not limited to, the presence of an induction period, an initial discussion of the role and frequency of supervision, one or two named clinical supervisors alongside other clinicians who support the PAs day-to-day work, clinical support that is initially more intensive but otherwise remains available when the PA feels it is required, and formal supervisory meetings that address clinical and pastoral components.

\section{References}

1 British Medical Association. Medical associate professions briefing. BMA, 2020. www.bma.org.uk/advice-and-support/nhs-deliveryand-workforce/workforce/medical-associate-professions-briefing [Accessed 12 November 2020].

2 NHS England, NHS Improvement. Network Contract Directed Enhanced Service: Additional Roles Reimbursement Scheme guidance. NHS, 2019. www.england.nhs.uk/wp-content/uploads/2019/12/ network-contract-des-additional-roles-reimbursement-schemeguidance-december2019.pdf [Accessed 14 November 2020].

3 Gowland B. The PCN Retention Challenge. Ockham, 04 November 2020. https://ockham.healthcare/blog-index [Accessed 15 November 2020].

4 Gopee N. Mentoring and supervision in healthcare, 2nd edn. SAGE Publications, 2011.

5 Hoskin J, Agarwal R. Preceptorship scheme for newly qualified physician associates working in general practice in Sheffield. Clin Med 2020;20:e255-9.

6 Faculty of Physician Associates. GP supervisor and physician associate guide: A step by step handbook for the first year in general practice. Royal College of Physicians. www.fparcp.co.uk/employers/ pas-in-general-practice [Accessed 14 November2020].

7 Drennan VM, Halter M, Brearley S et al. Corrigendum: Investigating the contribution of physician assistants to primary care in England: a mixed-methods study. Health Serv Deliv Res 2016:2:137-8.

8 Agarwal R, Mitchell S. Viewpoint: a proposed role for physician associates (PAs) in palliative care. BJGP Life, 02 November 2020 https://bjgplife.com/2020/11/02/viewpoint-a-proposed-role-forphysician-associates-pas-in-palliative-care [Accessed 12 November 2020].

9 Ritsema TS, Roberts KA. Job satisfaction among British physician associates. Clin Med 2016;16:511-3.

10 Kilminster S, Cottrell D, Grant J, Jolly B. AMEE Guide No. 27 . Effective educational and clinical supervision. Med Teach 2007;29: 2-19.

11 American Academy of PAs. How do PAs work with physicians? AAPA. www.aapa.org/what-is-a-pa/\#accordion__panel-2-how-dopas-work-with-physicians [Accessed 23 December 2020].

12 Scope of Practice Policy. Physician assistants overview. Scope of Practice Policy. http://scopeofpracticepolicy.org/practitioners/ physician-assistants [Accessed 12 November2020]

13 American Academy of PAs. What is a PA? AAPA, 2020. www. aapa.org/wp-content/uploads/2020/10/WhatIsAPA_Infographic_ LetterSize_October2020.pdf

Address for correspondence: Miss Ria Agarwal, Sothall Medical Centre, Eckington Road, Sheffield S20 1HQ, UK.

Email: riaagarwal19@googlemail.com

Twitter: @riaagarwal19 